\title{
Evidence Based Education e modelli di valutazione formativa per le scuole ${ }^{1}$
}

\author{
Antonio Calvani ${ }^{1}$ - Giuliano Vivanet ${ }^{2}$ \\ ${ }^{1}$ Università degli Studi di Firenze, Dipartimento di Scienze della Formazione \\ e dei Processi Culturali e Formativi \\ ${ }^{2}$ Università degli Studi di Cagliari
}

doi: 10.7358/ecps-2014-009-calv

antonio.calvani@unifi.it

giuliano.vivanet@unica.it

\section{EVIDENCE BASED EDUCATION \\ AND SCHOOL EDUCATION EVALUATION MODELS}

\begin{abstract}
Evidence Based Education (EBE) is emerging as an approach focusing on the problem of reliability in educational research, on its theoretical foundations, and on the relationship between research and decision-making. On the latter, less well known are the implications resulting from it for the development of innovative school evaluation systems based on dynamic methods, and thus differing from static models based on raw data compared to general standards. We shall firstly provide a brief summary of the EBE approach as well as the main research centers and general issues in this field. Then, we will show how notions of feedback and formative assessment, currently recognized as really effective individual learning strategies, can act as reference points for school evaluation and orientation models, by introducing the New Zealand system, developed with the collaboration of John Hattie, one of the most representative of the EBE approach, which suggests to move from a static to a dynamic accountability.
\end{abstract}

1 All'interno di una impostazione condivisa, di G. Vivanet sono i parr. 1 e 4, di A. Calvani i parr. 2 e 3 . Il contributo di G. Vivanet alla presente pubblicazione è stato prodotto durante l'attività di ricerca finanziata con le risorse del P.O.R. SARDEGNA F.S.E. 2007-2013 Obiettivo competitività regionale e occupazione, Asse IV Capitale umano, Linea di Attività 1.3.1 "Avviso di chiamata per il finanziamento di Assegni di Ricerca». 
Keywords: e-asTTle, Evidence based education, Feedback, Formative assessment, School evaluation.

\section{COS'È L'«EVIDENCE BASED EdUCATION»}

L'orientamento che va sotto il nome di Evidence Based Education (EBE), espressione poi ammorbidita in Evidence Informed Education o Evidence Aware Education, prende sviluppo a partire dall'inizio degli anni '90, diffondendosi in particolare nella cultura pedagogica anglosassone (cfr. Form@re, 2013; Vivanet, 2014).

Si tratta di un movimento culturale che sta sempre più pervadendo il mondo dell'educazione e che si caratterizza, pur nella diversità delle posizioni (cfr. Hammersley, 2007), per alcuni lineamenti d'insieme che saranno di seguito sinteticamente richiamati.

In primo luogo, vi è l'idea che ogni ricerca che aspiri a un impatto sociale, dunque anche in ambito educativo, debba passare attraverso una completa esplicitazione delle proprie assunzioni valoriali e scientifiche (Calvani, 2012). Inoltre, l'EBE, nel suo impegno a favore di una conoscenza affidabile e spendibile nelle politiche e nelle pratiche operative, è contrassegnato da un sostanziale atteggiamento critico nei riguardi della ricerca educativa tradizionale (Vivanet, 2013). In particolare, di quest'ultima si sottolinea sia il carattere eccessivamente ideologico, tratto dal fatto che le ricerche spesso assomiglino più a "dichiarazioni di fede» che a resoconti scientifici, sia la distanza dalla pratica, e la conseguente incapacità di offrire risposte concrete alle questioni rilevanti nella didattica quotidiana (Coe, 1999; Davies, 1999; Slavin, 2004; Hargreaves, 2007).

La diffusione dell'EBE è coadiuvata attualmente e si svolge in concomitanza con diversi processi, tra i quali appaiono particolarmente degni di attenzione i seguenti: (i) l'arricchimento considerevole dei risultati della ricerca educativa; (ii) le crescenti esigenze e richieste dei decisori politici nei riguardi di quest'ultima; e (iii) il ruolo sempre più importante del Web nella diffusione delle conoscenze didattiche.

Con riferimento al primo punto, stiamo assistendo al moltiplicarsi dei contributi della ricerca educativa sia sul versante delle comparazioni dei siste$\mathrm{mi}$ di istruzione, tra le più significative quelle derivanti dai programmi PISA ed Education at a glance dell'OECD, TIMSS della IEA e The learning curve della Pearson, sia su quello delle sintesi di conoscenze e bilanci critici circa l'efficacia di metodologie didattiche, con nuove possibilità che si prospettano per una scienza dell'istruzione fondata su evidenza (Marzano, Pickering, \& 
Pollock, 2001; Mayer, 2005; Mitchell, 2007; Hattie, 2009; Clark \& Lyons, 2010; Mayer \& Alexander, 2010; Clark, Nguyen, \& Sweller, 2011).

Relativamente al secondo punto, la richiesta da parte dei decisori (quali dirigenti scolastici e policy makers) nei riguardi della ricerca educativa si è fatta più esigente per le nuove sfide con cui l'istruzione si deve confrontare, tra cui: comparazioni internazionali, introduzione di nuove tecnologie, inclusione, cittadinanza, nuove competenze, lifelong learning. Di conseguenza, sul piano delle politiche è oggettivamente sempre più difficile giustificare finanziamenti ad ambiti di studio che avanzano pretese di rilevanza e accreditamento sociale, rivelandosi poi elusivi sul piano della rendicontazione. Al riguardo, è emblematico il caso del No child left behind Act (U.S. DoE, 2002), provvedimento di riforma del sistema di istruzione americano, la cui attuazione è stata accompagnata da un acceso dibattito, incentrato sui dati di valutazione del suo impatto socio-educativo, in cui sono state evidenziate numerose criticità quali: l'impoverimento del curriculum degli studenti, finalizzato al superamento del test; la riduzione di impegno nei confronti delle aree disciplinari che non sono oggetto dei test; la scarsa attenzione agli studenti con bisogni educativi speciali, alle cui esigenze un modello valutativo rigidamente standardizzato non può venire incontro (cfr. Holland, 2004; Jennings \& Rentner, 2006; Frontline, 2013; Parents for Choice in Education, 2013; Smith, 2013).

Con riferimento al terzo punto, insegnanti e professionisti della formazione hanno oggi possibilità, inimmaginabili solo pochi anni fa, di accesso alla conoscenza scientifica e alle dimostrazioni operative connesse alle diverse metodologie didattiche, grazie alla evoluzione del Web, caratterizzata dai cambiamenti connessi allo sviluppo tecnologico dei motori di ricerca (Google e Google Scholar in particolare); alla diffusione della cultura open access/content; alla crescente disponibilità di repertori di conoscenze e casi didattici, documentati anche visivamente, specificamente dedicati alla formazione degli insegnanti (quali YouTube for Teachers e Teaching Channel) ${ }^{2}$.

In tale quadro, il crescente sviluppo dell'EBE è alimentato dall'attività di numerosi istituti di ricerca, organizzazioni no-profit e associazioni (cfr. Bruni \& Vivanet, 2013), oltre che dalla disponibilità di risorse documentarie (Figura 1), il cui principale scopo è stabilire raccordi più stretti tra ricerca $\mathrm{e}$

2 Quest'ultimo aspetto, in particolare, pone nuove istanze per i professionisti della formazione, come evidenziato da numerosi organizzazioni internazionali (cfr. ACRL, 2000; Parlamento Europeo e del Consiglio, 2006; UNESCO, 2008): infatti, la sempre crescente disponibilità di risorse digitali utili per le professionalità educative richiede adeguate competenze informative (information literacy) per avere loro accesso e per la loro selezione (Bonaiuti \& Vivanet, 2013). 
pratiche didattiche, vuoi partendo dal versante della ricerca verso le pratiche (modelli di didattica efficace), vuoi nel senso contrario (pratiche basate su evidenze, buone pratiche, ecc.).

Tra gli istituti di ricerca internazionali più attivi in questo ambito, si segnalano per primi il What Works Clearinghouse (WWC) negli Stati Uniti e l'Evidence for Policy and Practice Information and Co-ordinating Centre (EPPI-Centre) nel Regno Unito, entrambi impegnati nella produzione, attraverso analisi e sintesi di ricerca, e diffusione, tramite banche dati liberamente consultabili on-line, di conoscenze utili per rafforzare una cultura pedagogica informata e consapevole delle evidenze. Oltre a questi, si ricordano il Center for Research and Reform in Education, interessato principalmente alla fascia K-12; l'Institute for Effective Education dell'Università di York; e il Top Institute for Evidence Based Education Research, un'iniziativa congiunta delle Università di Amsterdam, Maastricht e Groningen.

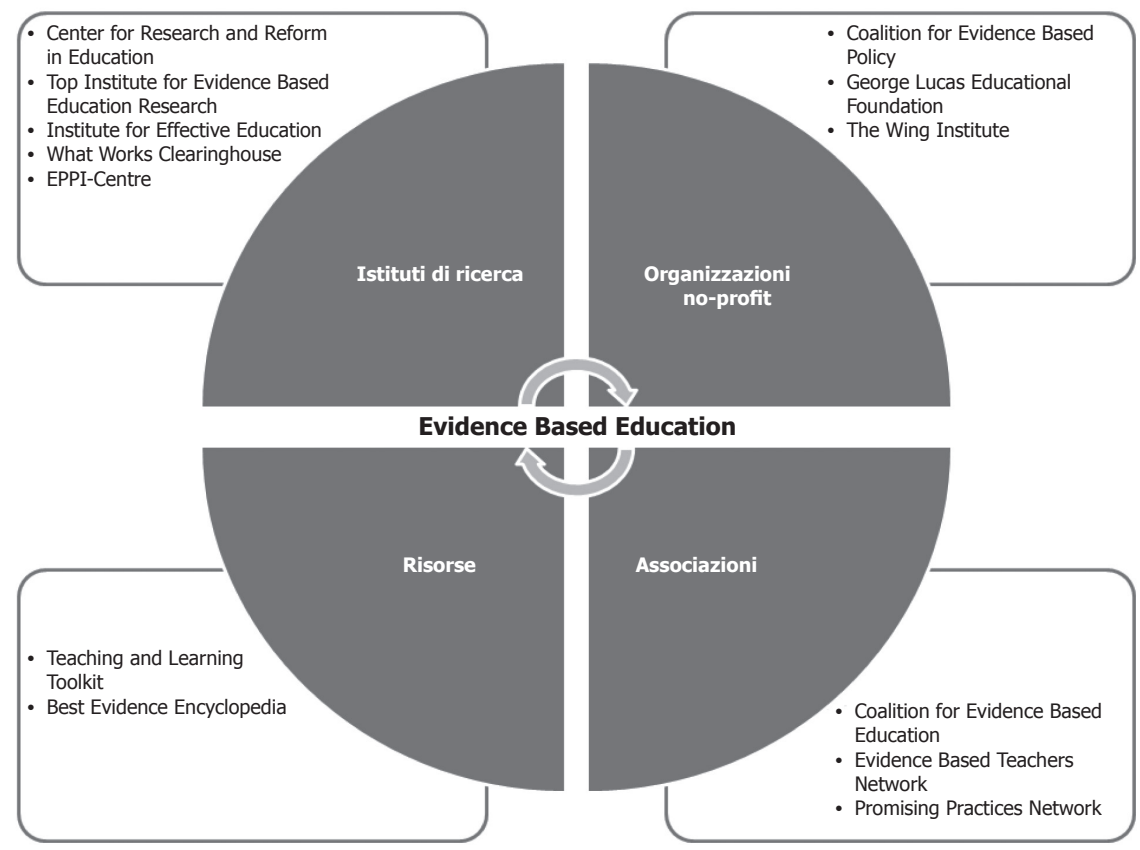

Figura 1. - Centri attivi nel mondo dell'Evidence Based Education, suddivisi per tipologie (cfr. Bruni \& Vivanet, 2013). 
Numerose sono anche le organizzazioni no-profit attive in questo ambito. Tra esse, il The Wing Institute, organizzazione indipendente attiva nella promozione di politiche e pratiche educative basate sui risultati della ricerca empirica; la George Lucas Educational Foundation, curatrice di Edutopia, un ambiente on-line ricco di risorse rivolte specialmente a educatori e insegnanti della formazione primaria e secondaria; e la Coalition for Evidence-Based Policy, impegnata in politiche educative per la prevenzione della criminalità.

Si segnala, inoltre, l'esistenza di diverse associazioni di professionisti del mondo della formazione il cui obiettivo è condividere le conoscenze emergenti in seno all'EBE, quali: l'Evidence Based Teachers Network; la Coalition for Evidence-Based Education; e la Promising Practices Network.

Infine, tra le risorse documentarie disponibili, si ricordano il Teaching and learning toolkit, curato dall'Education Endowment Foundation, un agevole strumento di consultazione che riporta indicazioni circa l'efficacia di una trentina di opzioni didattiche; e la Best evidence encyclopedia, archivio di risorse destinato a educatori e ricercatori per la diffusione di evidenze emergenti nei programmi di istruzione per studenti K-12.

\section{2. «EVIDENCE BASED EDUCATION»: NODI CRITICI}

La rilevanza che l'EBE sta assumendo nel mondo dell'educazione si accompagna a un complesso dibattito che vede schierate, in uno spettro articolato, posizioni che spaziano dal rifiuto pregiudiziale, proprio di chi muove da posizioni fenomenologiche radicali, all'accettazione di una versione più intransigente di questo orientamento da parte di chi vede per l'educazione perseguibile la strada di una capitalizzazione progressiva della conoscenza, in modo non dissimile da quanto è accaduto per la medicina (Slavin, 2004; Hargreaves, 2007).

Senza entrare qui nel merito di tali posizioni, ci limitiamo a sottolineare che sarebbe semplicistico vedere nell'EBE una sorta di rinato neopositivismo con le comuni attribuzioni che a questo concetto normalmente si associano, soprattutto nella sua versione più ingenua (primato dei fatti, messa al bando di metodologie non rigorosamente sperimentali o addirittura delle stesse idee-ideologie) (Calvani, 2013) ${ }^{3}$. Una posizione del genere ignorerebbe la riflessione critica che questo movimento sta sollevando e la consapevolezza

3 Dopo Dewey, Popper e Kuhn e le speculazioni da essi derivate, chiunque opera nell'ambito della ricerca non può che essere ben consapevole che una nuova conoscenza non può emergere da un puro accumulo quantitativo di dati; né che si possa pervenire ad una conoscenza esaustiva e incontrovertibile. 
epistemologica che lo accompagna. È ormai sufficientemente condiviso che la ricerca sul "Cosa funziona» (What works) non appare significativa se non accompagnata da una attenta valutazione delle circostanze specifiche caratterizzanti i singoli contesti didattici (under what circumstances) (Pawson \& Tilley, 2004) e dei limiti legati alla trasferibilità delle conoscenze acquisite ${ }^{4}$.

Anche la recente preferenza terminologica per termini come Informed o Aware Education (al posto del Based) rivela la consapevolezza di questo delicato problema e intende rimarcare l'abbandono di ogni deduzionismo meccanico: lo scopo della ricerca è mettere sul tavolo le conoscenze acquisite all'interno di contesti di studio in qualche misura assimilabili e di informare o rendere consapevole il decisore di questi risultati, non di prescrivere il da farsi. Il decisore, una volta che conosca l'esistenza di possibilità alternative rispetto a quelle cui lui stesso aveva pensato, potrà gestire la sua scelta anche in senso difforme o contrario al criterio/modello suggerito dalla ricerca, tenendo conto di eventuali altri fattori (sociali, culturali, ecc.) considerati di maggiore rilevanza. Per fare un semplice esempio, l'acquisizione, risultante da numerose indagini, secondo cui l'uso dei compiti a casa nella scuola primaria non migliora l'apprendimento non significa che si debba automaticamente rinunciare a questi ultimi; una simile conoscenza, invece, potrebbe stimolare negli insegnanti e nelle famiglie una comune ricerca per una funzione diversa da assegnare a tali compiti (Hattie, 2012).

In breve, la conoscenza scientifica non deve calare dall'alto, soffocando saggezza pratica e sensibilità verso il contesto socio-culturale, ma anzi deve aiutare a dare loro "voce», contribuendo in tal modo al conseguimento di un'effettiva competenza esperta. Come scrive Hargreaves (2007, p. 12): «Expertise non significa solo avere rilevante esperienza e conoscenza ma anche competenza dimostrabile e chiara evidenza in grado di giustificare perché si fanno le cose in un modo anziché in un altro».

\section{3. «EVIDENCE BASED EDUCATION»: IMPLICAZIONI DIDATTICHE}

Se ci chiediamo in che senso questo orientamento possa incidere sulla cultura e sulle pratiche dell'educazione, per comodità di analisi possiamo individuare tre diverse implicazioni del concetto di evidenza, che chiameremo epistemolo-

4 In un precedente lavoro su questa rivista uno degli scriventi ha sviluppato queste problematiche esprimendo anche la sua preferenza per una accezione epistemologica avveduta della "capitalizzazione delle conoscenze» vista come focalizzazione di sistemi di «conoscenze sfidanti»(Calvani, 2011b). 
gica, tecnica, e didattica. In un'accezione epistemologica più generale, il concetto di evidenza può essere identificato con un processo conoscitivo capace di esplicitarsi, cioè di rendere trasparente e ripetibile il processo attraverso il quale da una ipotesi o domanda originaria, si è pervenuti a una determinata conclusione. Si cerca così di eliminare quanto più possibile interferenze e tendenziosità (ideologiche, linguistiche e/o strumentali), consentendo così la falsificabilità dell'ipotesi (Popper, 1972; Kuhn, 1979).

Questo aspetto ha interessanti implicazioni per l'educazione, in quanto agisce come richiamo a un maggiore controllo sulla qualità del linguaggio impiegato, a una riduzione della genericità e ambiguità in esso presente, e a un avvicinamento del linguaggio pedagogico alle caratteristiche di trasparenza ed esplicitezza proprie di quello scientifico.

In un'accezione tecnica, di interesse più specifico per chi fa ricerca, la nozione di evidenza sottolinea la necessità di differenziare e stabilire gerarchie tra le varie metodologie in funzione della loro capacità di garantire livelli differenti di affidabilità. Così, in genere, le meta-analisi sono ritenute di maggiore affidabilità rispetto a una singola ricerca sperimentale; a sua volta, una ricerca sperimentale con gruppo di controllo è ritenuta più affidabile di un'indagine quasi-sperimentale; in ambito qualitativo, l'impiego di triangolazioni aumenta l'affidabilità della ricerca e cosi via.

Lo scopo che ci si propone da questo punto di vista è acquisire contenuti conoscitivi capitalizzabili, in modo simile a quanto accade nella medicina, rendendo pertanto possibili sintesi affidabili della conoscenza relativa al dominio e, dunque, pervenire a uno «stato dell'arte». Al di là della sostenibilità di una analogia «stretta" con il campo medico, lo sforzo di fare il punto su quanto si sa, in merito a un determinato quesito o argomento, rappresenta una significativa sollecitazione per la ricerca educativa.

Da un lato questa è indotta a mettere a nudo le ambiguità e le false credenze; per fare un esempio, gli stili di apprendimento hanno rappresentato per molti anni una sorta di mitologia a cui non corrispondono esempi ed evidenze convincenti in termini di efficacia (Hattie, 2009; Clark \& Lyons, 2010; Calvani, 2011a, 2012; Gruber, 2011) e discorso analogo si può fare sulle conoscenze (o meglio credenze) che si hanno sull'efficacia delle tecnologie, sistematicamente sconfessate dalle evidenze scientifiche (Calvani, 2012). Dall'altro le conoscenze che risultano di maggiore affidabilità possono rappresentare benchmark, assunti per perimetrare il territorio, in una dialettica con nuove "conoscenze sfidanti» capaci di metterle in discussione o di favorire correzioni o riformulazioni più articolate (Calvani, 2011b).

In un'accezione didattica, l'EBE si caratterizza per il suo intento di rendere la conoscenza spendibile sul terreno operativo, traducendosi in indicazioni e azioni che rendono chiaro come conseguire un miglioramento 
concreto. All'educatore interessa quanto la conoscenza ha da dire sul piano dell'intervento effettivo; in breve, tale accezione riguarda il "come si debba operare per migliorare l'efficacia degli interventi didattici». Tuttavia, se ci spostiamo dal livello della classe a quello più ampio delle politiche educative, le ricerche in ambito EBE sono per lo più identificate come una sorta di impalcatura a supporto dell'impiego di standard e traguardi generali per la valutazione scolastica, in un'ottica generalmente statica e classificatoria.

Contro questa concezione si è mosso John Hattie, direttore del Melbourne Education Research Institute presso l'Università di Melbourne e uno dei maggiori rappresentanti dell'EBE contemporanea. Egli ha dedicato gran parte del suo lavoro a definire lo stato dell'arte sulle metodologie didattiche efficaci, riportando a sintesi, su una scala di Effect Size (ES) ${ }^{5}$, dati emergenti da oltre ottocento meta-analisi, al fine di evidenziare i fattori che influenzano i risultati degli studenti in età scolastica (Hattie, 2009, 2012; Hattie \& Anderman, 2013). La sua analisi si è concentrata su circa centocinquanta fattori riconducibili a sei aree di influenza così definite: lo studente, l'ambiente domestico, l'ambiente scolastico, l'insegnante, il curriculum, la strategia di insegnamento.

Dalla sua sintesi risulta centrale il concetto di visible teaching-learning, inteso quale processo visibile ed esplicito, supportato da un continuo feedback nella relazione insegnante-allievo e dal momento valutativo quale strategia determinante per il raggiungimento degli obiettivi didattici; l'uso combinato della valutazione formativa (ES 0.90) e del feedback (ES 0.75) contribuisce significativamente al miglioramento dei risultati degli studenti (Hattie \& Timperley, 2007; Hattie, 2009, 2012) ${ }^{6}$.

Muovendo dalle evidenze empiriche relative alla efficacia di una didattica in classe basata sul feedback e sulla valutazioen formativa ${ }^{7}$, Hattie (2013) prende anche posizione nei confronti di quella filosofia della valutazione basata sull'uso di traguardi nazionali e di sistemi di premi e punizioni che

5 L'Effect Size è un indice che esprime la dimensione di un effetto, in questo caso l'efficacia didattica di un fattore. Più alto il suo valore e più la strategia didattica è risultata efficace. Si considera generalmente significativo un valore di efficacia oltre 0.40 .

6 Come noto, il riconoscimento del valore della valutazione formativa ha una lunga tradizione nella ricerca educativa, a partire dalla distinzione classica operata da Scriven (1967) tra formative evaluation e summative evaluation, passando attraverso la proposta di Bloom (1968) del mastery larning, fino a tempi più recenti con le evidenze sulla sua efficacia emergenti nella meta-analisi condotta da Black e Wiliam (1998).

7 Parlare di una didattica centrata sul feedback non autorizza a dedurre una formuletta del tipo «tanto più feedback l'insegnante fornisce tanto più apprendimento si produce». Il problema è più complesso. ci sono anche feedback negativi; un buon feedback deve comunque mostrare "dove sto andando, come sto procedendo, quale deve essere il prossimo passo" (Hattie, 2009; Hattie \& Gan, 2011). 
gravano sulle scuole dall'esterno, esprimendosi invece a favore di soluzioni che attribuiscono il controllo del processo valutativo a insegnanti e scuole (come si vedrà nel paragrafo seguente).

Importante è l'atteggiamento dell'insegnante verso la valutazione: questi non comprende come essa sia parte integrante dell'apprendimento. Allo stesso tempo una prova esterna che giudica i risultati della propria classe attiva meccanismi psicologici di ansia e di difesa nell'insegnante, con comportamento di cheating (ad esempio, non far partecipare alla prova alunni con difficoltà). Se poi l'insegnante riceve un feedback che evidenzia un gap rispetto alla situazione nazionale, tenderà a difendersi e autogiustificarsi disconoscendo la qualità della prova (ad esempio, accusando quest'ultima di essere una forma di imposizione ideologica o di controllo insensibile alle specificità locali).

A giudizio di Hattie occorre ricercare strade che da un lato prevedano anche una politica di maggiore flessibilità e supporto orientativo esercitato dagli organi centrali alle scuole e dall'altro una maggiore responsabilizzazione di insegnanti e scuole nei confronti della valutazione e dei suoi traguardi: l'attenzione va spostata dal risultato statico al test al miglioramento e ai modi più adeguati per metterlo in chiara evidenza e renderlo oggetto di apprezzamento.

Abbiamo bisogno di politiche che rendano le scuole unità di valutazione e di aiutare ogni scuola a lavorare collaborativamente per definire i risultati che intende valutare. Bisogna aiutare le scuole a raccogliere evidenze affidabili dei livelli correnti e di quelli desiderati e monitorare criticamente i progressi. Ancora più importante che ci sia riconoscimento e stima quando questi progressi sono compiuti e i successi devono essere resi pubblici nella comunità scolastica. (Hattie 2012, p. 168)

Molte di queste istanze si incarnano nel modello che Hattie ha contribuito a implementare in Nuova Zelanda e che sarà di seguito illustrato.

\section{COME INTEGRARE EVIDENZA E VALUTAZIONE}

NEL PROCESSO FORMATIVO:

L'ESPERIENZA DEL MODELLO E-ASTTLE

(«Assessment TOOLS FOR TEACHING AND LEARNING»)

L'interesse per il modello valutativo neozelandese (similmente adottato anche in Australia) è qui riconducibile a due principali ragioni: gli spunti di riflessione che questo può offrire in relazione al dibattito in corso nel no- 
stro paese sul tema della valutazione nella scuola e il fatto che esso sia ben esemplificativo della traduzione dei risultati della ricerca EBE in decisioni e pratiche didattiche (in Nuova Zelanda, infatti, l'approccio evidence-based ha avuto, nell'ultimo decennio, una notevole influenza nell'informare la politica educativa). Sullo sviluppo di tale sistema ha avuto grande influenza il lavoro già citato di Hattie e, in particolare, le ricerche sull'efficacia didattica dell'uso combinato di adeguate strategie di valutazione formativa e opportuni feedback tra insegnante e studente (da intendersi in entrambe le direzioni).

Il sistema scolastico neozelandese ha tradotto tali indicazioni di ricerca in un modello di valutazione che mette al centro le finalità formative di quest' ultima ${ }^{8}$. Esso si caratterizza per il tentativo in atto di contemperare due istanze apparentemente divergenti: (i) la necessità di standardizzazione dei curriculum scolastici in modo da garantire il raggiungimento di livelli base di conoscenze e competenze da parte di tutti gli studenti e (ii) quella di garantire la flessibilità del sistema valutativo stesso al fine di corrispondere alle finalità sia formative sia inclusive delle singole realtà scolastiche.

La prima di tali istanze trova risposta nella recente formulazione del National curriculum che identifica le direzioni verso cui deve tendere il sistema scolastico (quali valori, quali competenze, quali principi, in quali aree disciplinari) e una guida per la traduzione di queste nei singoli curriculum scolastici, attraverso l'identificazione di obiettivi e strategie didattiche efficaci. Sulla base del curriculum nazionale, sono stati poi definiti, per gli alunni tra i 5 e i 12 anni, i National standards che fungono da linee guida per la definizione delle competenze per specifiche aree (lettura, scrittura e matematica).

Tale standardizzazione si declina flessibilmente nelle singole realtà scolastiche, garantendo un'ampia libertà e spazio di autonomia alle scuole e agli insegnanti nella definizione dei propri obiettivi didattici e dei metodi di valutazione. A livello delle istituzioni scolastiche (singola scuola o reti di scuole), è previsto si attui un processo, detto moderation, durante il quale gli insegnanti si impegnano collegialmente nella definizione dei percorsi didattici e nella definizione condivisa delle modalità di valutazione del processo di apprendimento dei propri studenti, in relazione agli standard nazionali (quali evidenze considerare e quali criteri utilizzare per la loro interpretazione). Questo processo ha tra i suoi obiettivi anche quello di ottenere una

8 Il Ministero dell'Educazione neozelandese esprime la propria visione della valutazione degli apprendimenti in un documento ufficiale, denominato The Ministry of Education position paper: Assessment (New Zealand Ministry of Education, 2011), in cui ne sono esplicitati i riferimenti teorici, motivazioni e obiettivi. Nell'ottica di costruire una condivisione della filosofia valutativa adottata, lo stesso Ministero ha predisposto un apposito ambiente Web indirizzato a chi opera nelle scuole, agli studenti e alle famiglie. URL: http://assessment.tki.org.nz. 
certa uniformità di valutazione tra i diversi docenti e adattare i processi di insegnamento e di valutazione alle specificità dei singoli contesti scolastici.

A livello del docente, tale flessibilità si concretizza nella formulazione da parte di quest'ultimo del proprio giudizio complessivo sul processo di apprendimento dello studente, l'Overall Teacher Judgement (OTJ) che il docente può definire attraverso i metodi e gli strumenti di valutazione, formali e informali, che ritiene più adeguati per il proprio specifico contesto. In tal senso, le indicazioni nazionali rappresentano più delle linee guida entro cui scuole e docenti devono individuare gli obiettivi adeguati per il proprio contesto, che norme da applicare rigidamente e indipendentemente da qualsiasi specificità. Nell'espressione della propria valutazione, secondo quanto indicato dal Ministero dell'Educazione neozelandese, ogni insegnante deve tenere conto di differenti dati, triangolando vari tipi di evidenze, in quanto una singola fonte di informazioni non può essere adeguata a rappresentare esaustivamente la complessità del processo di apprendimento. Tali evidenze, quantitative e qualitative, sono riconducibili a tre macro-dimensioni: (i) l'osservazione dello studente (ad esempio, nei momenti di svolgimento dei compiti, delle interazioni tra pari, della relazione coi docenti); (ii) il dialogo con lo studente (ad esempio, mirato a rilevare le conoscenze e competenze apprese, gli atteggiamenti, le percezioni, l'auto-valutazione); (iii) i risultati ottenuti in valutazioni formali (ad esempio, somministrazione di test standardizzati). I diversi elementi rilevati, inoltre, devono essere messi in relazione a informazioni sul contesto scolastico (quali strutture, orari, organizzazione, risorse) e sociale (quali etnia, genere, età, fattori occupazionali, famiglia e comunità), in cui il processo di apprendimento è situato, data l'influenza che questo può avere sugli esiti di quest'ultimo.

Attraverso la combinazione di evidenze qualitative e quantitative, formali e informali, si cerca di limitare i rischi connessi all'adozione di sistemi valutativi centrati fortemente sull'uso di test standardizzati, quali quelli precedentemente citati in relazione al No child left behind Act. Di fronte a simili rischi, la stessa adozione dei test standardizzati nel contesto neozelandese presenta elementi peculiari. Gli insegnanti e le scuole, infatti, hanno piena autonomia nel decidere se e quali strumenti di valutazione in itinere utilizzare, in base all'adeguatezza di questi ultimi rispetto ai propri particolari obiettivi di istruzione. A tal fine, il Ministero dell'Educazione ha predisposto un apposito ambiente Web, denominato Assessment tool selector ${ }^{9}$, che consente di confrontare e selezionare circa ottanta differenti strumenti di valutazione gratuiti (resi disponibili dallo stesso Ministero o forniti da università o terze parti) sulla base dell'area disciplinare e delle eventuali relative sub-competenze (es. inglese: lettura, scrittura, spelling, ecc.); del livello scolastico; della corrispon-

9 URL: http://toolselector.tki.org.nz. 
denza col curriculum neozelandese; del fatto che sia standardizzato o no; o che sia progettato per la valutazione di gruppi o di singoli studenti.

Uno degli strumenti più diffusi, soprattutto nelle scuole primarie neozelandesi, come rilevato in un recente Report (Nusche et al., 2012) dell'Organisation for Economic Co-operation and Development (OECD), è e-asTTle (e-Assessment Tool for Teaching and Learning) ${ }^{10}$, sviluppato nell'ambito di un progetto guidato dallo stesso Hattie, in collaborazione con la Università di Auckland. Si tratta di un tool per la valutazione on-line degli apprendimenti nella lettura, scrittura e matematica, pensato esplicitamente per valorizzare il momento valutativo in chiave formativa. Una caratteristica fondamentale di e-asTTle è il fatto che i test generati dal sistema (nella forma di item a risposta sia chiusa sia aperta), calibrati sugli standard nazionali, possono essere comunque adattati dall'insegnante affinché siano coerenti con gli obiettivi didattici e il percorso di apprendimento della propria classe, ad esempio ridefinendo il grado di difficoltà, anche per singoli studenti, sulla base dei risultati di precedenti valutazioni.

Scopo della valutazione, secondo il modello di e-asTTle, dovrebbe essere quello di comunicare e condividere chiaramente, in primis con gli studenti ma anche con le famiglie: (i) il punto in cui si trova lo studente all'interno del percorso didattico; (ii) l'obiettivo finale da raggiungere; e (iii) la strada per raggiungere quest'ultimo, attraverso degli obiettivi intermedi.

e-asTTle, in tal modo, intende fornire un feedback realmente formativo, relativo al progresso dello studente, evidenziandone aree di forza e punti di debolezza, rappresentati e sintetizzati attraverso uno schema a quattro quadranti che indicano quanto segue (Figura 2):

- Obiettivi raggiunti (Achieved): item, corrispondenti a obiettivi in linea con gli standard nazionali, a cui lo studente, considerato il livello in cui si trova nel curriculum, avrebbe dovuto rispondere correttamente e a cui effettivamente è stata data risposta corretta. Questa informazione può suggerire all'insegnante che lo studente ha raggiunto, nelle aree interessate, una sufficiente padronanza e, pertanto, la possibilità di prevedere compiti futuri più impegnativi.

- Punti di forza (Strengths): item, corrispondenti a obiettivi in linea con gli standard nazionali, a cui lo studente, considerato il livello in cui si trova nel curriculum, non avrebbe dovuto rispondere correttamente e a cui, invece, è stata data risposta corretta. Questa informazione deve essere analizzata dall'insegnante per comprendere se le risposte corrette sono frutto di tentativi di risposta casuali o, in caso contrario, se rappresentano aree di poten-

10 Si tratta di una evoluzione del precedente modello, denominato asTTle, inizialmente distribuito su CD-Rom gratuitamente alle scuole. Il sito ufficiale è http://e-asttle.tki.org.nz. 
ziale sviluppo per lo studente di cui tenere conto nel prosieguo del processo di insegnamento-apprendimento.

- Lacune (Gaps): item, corrispondenti a obiettivi in linea con gli standard nazionali, a cui lo studente, considerato il livello in cui si trova nel curriculum, avrebbe dovuto rispondere correttamente e a cui, invece, è stata data una risposta scorretta. Questa informazione richiede grande attenzione e deve essere analizzata dall'insegnante per individuare le ragioni degli errori, se attribuibili ad esempio allo studente (quali disattenzione o problemi personali) o all'insegnamento e prendere di conseguenza iniziative appropriate.

- Obiettivi da raggiungere (To be achieved): item, corrispondenti a obiettivi in linea con gli standard nazionali, a cui lo studente, considerato il livello in cui si trova nel curriculum, avrebbe dovuto rispondere scorrettamente e a cui effettivamente è stata data una risposta scorretta. Questa informazione suggerisce all'insegnante gli obiettivi cui tendere nel prosieguo dell'insegnamento.

Risposte corrette

\section{Punti di forza}

Identificare e comprendere i concetti chiave: (3:5)

- Punteggiatura: (1:7)

- Usare correttamente le strutture grammaticali: (1:9)

-

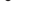

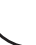

\section{Obiettivi raggiunti}

- Trovare e selezionare le informazioni: $(2: 6 ; 3: 2)$

- Fare uso delle preconoscenze: $(2: 1)$

- ...
Risposte scorrette

\section{Obiettivi da raggiungere}

Comprendere e interpretare accuratamente le informazioni: (1:8)

- Identificare le caratteristiche del linguaggio in testi storici: (3:6)

- ... 
In tal senso, ciò che è veramente rilevante nella valutazione compiuta con e-asTTle non è tanto il dato valutativo in sé, espresso ad esempio dal numero di risposte corrette fornite, quanto l'interpretazione dei dati, utile per guidare il percorso formativo seguente. In aggiunta, sulla base di tali indicazioni, il sistema suggerisce la fruizione di specifiche risorse didattiche mirate al raggiungimento di obiettivi in linea con gli standard nazionali ${ }^{11}$.

Infine, le informazioni prodotte possono rappresentare utili elementi informativi anche per dirigenti scolastici e amministratori nell'assunzione di decisioni più consapevoli, in quanto tra le diverse funzionalità offerte, e-asTTle restituisce un quadro comparativo dei dati di ogni singolo studente, classe e scuola rispetto, ad esempio, alle medie nazionali, e ai dati per genere, età, etnia e così via.

Non manca, tuttavia, in letteratura (Thrupp \& White, 2013; Wylie \& Berg, 2013) il richiamo ai rischi conseguenti all'introduzione degli standard nazionali a fondamento del sistema scolastico neozelandese, quali: l'adozione di metriche standardizzate potrebbe rispondere più alle esigenze politiche di «rendicontazione» che ai bisogni formativi degli studenti; la definizione degli standard nella lettura, scrittura e matematica potrebbe rendere marginali aree differenti; dirigenti scolastici e insegnanti sarebbero sovraccaricati di lavoro, dato il considerevole impegno richiesto per il processo di valutazione, senza aver ricevuto un'adeguata preparazione professionale né adeguati riconoscimenti.

Allo stesso tempo, in una recente indagine dell'OECD (Nusche et al., 2012) ${ }^{12}$ si manifesta un sostanziale apprezzamento per una politica valutativa esplicitamente orientata al miglioramento degli apprendimenti (tale enfasi sarebbe ridotta nella istruzione secondaria), oltre che per la responsabilizzazione professionale del docente nella definizione della strategia di valutazione e per la disponibilità di differenti strumenti a supporto degli insegnanti a tale scopo.

La stessa indagine evidenzia le sfide più importanti di fronte a cui si trova il sistema di valutazione neozelandese, e che in qualche modo rappresentano i nodi cui, nel suo sviluppo futuro, bisogna prestare attenta considerazione, quali: la definizione e il ruolo di standard nazionali in rapporto

11 Il sistema è, infatti, arricchito di un archivio di risorse didattiche, denominato What next, raggiungibile al seguente indirizzo: http://assessment.tki.org.nz/Assessment-tools-resources/ What-Next.

12 L'indagine citata è parte di un Progetto di ricerca comparativa internazionale più ampia, denominata "OECD review on evaluation and assessment frameworks for improving school outcomes", il cui obiettivo è comprendere come i sistemi di valutazione possano migliorare la qualità, l'equità, e l'efficienza dei sistemi scolastici. URL: http://www.oecd.org/education/ school/oecdreviewonevaluationandassessmentframeworksforimprovingschooloutcomes.htm 
agli obiettivi del sistema scolastico; il ruolo dei docenti nella valutazione e la loro formazione professionale; la necessità di contemperare istanze valutative e istanze inclusive; l'equilibrio tra le esigenze di uniformità e condivisione del sistema valutativo da una parte e la necessità di garantire la libertà del docente nella espressione del proprio giudizio dall'altra (Nusche et al., 2012).

Alla luce di quanto fin qui esposto, e-asTTle e, più in generale, il modello neozelandese, trasferendo indicazioni evidence-based in politiche e pratiche didattiche, rappresentano un interessante oggetto di analisi, anche in relazione al dibattito in corso nel nostro paese sulla valutazione degli apprendimenti e dei sistemi scolastici. In proposito, alcuni aspetti meritano di essere sottolineati:

- la sostenibilità di un modello valutativo quale quello neozelandese è possibile laddove ci si impegni nella formazione di una cultura della valutazione, in cui il momento valutativo è accolto quale occasione di sviluppo per il sistema stesso e di apprendimento per gli studenti e in cui vi sia confronto e condivisione con gli attori del sistema scolastico (quali dirigenti, insegnanti, studenti e famiglie);

- un sistema di valutazione che si basi esclusivamente sulla definizione di strumenti di valutazione e standard nazionali rischia di condizionare i percorsi curricolari favorendo una cultura educativa dipendente dal superamento del test;

- la valutazione dell'efficacia di un sistema scolastico nella formazione dei propri studenti non può esaurirsi nella registrazione di dati derivanti da test nazionali e internazionali standardizzati e relative comparazioni; occorre integrare con altri strumenti rispondenti alle specificità locali;

- si deve evitare di creare sistemi che generano grandi quantità di dati a cui non corrispondono quasi mai precise indicazioni di cambiamento;

- uno dei rischi maggiori generati dai sistemi standardizzati di valutazione è quello di rendere di fatto i soggetti con bisogni educativi speciali (qualsiasi sia la loro origine) estranei alla valutazione stessa, in quanto i loro obiettivi didattici non possono rientrare in quadri definiti a livello nazionale; la definizione di un sistema di valutazione degli apprendimenti scolastici non può ignorare le sue implicazioni sui processi di inclusione sociale;

- infine, il sistema di valutazione richiede la disponibilità di adeguate risorse per garantire da un lato la preparazione professionale di dirigenti e insegnanti e dall'altro la disponibilità di adeguati strumenti, anche tecnologici, di supporto. 


\section{CONCLUSIONI}

L'Evidence Based Education è un orientamento culturale che ha fondamentalmente lo scopo di superare lo iatus tradizionalmente esistente tra la teoria e la pratica educativa. Per comodità abbiamo distinto in esso tre principali implicazioni, una più epistemologica, una più tecnica, una più didattica. Sul piano strettamente didattico l'EBE si occupa di fornire agli insegnanti suggerimenti relativi ai metodi più efficaci. In questo ambito essa investe anche le problematiche della valutazione, dato l'alto livello di efficacia che la valutazione formativa e l'uso appropriato del feedback conseguono tra tutte le azioni didattiche.

In questo contributo ci si è chiesti se da questo movimento emergano anche indicazioni e modelli relativi all'attuazione di nuovi sistemi di valutazione delle scuole. Uno dei più noti rappresentanti della ricerca evidence-based contemporanea, John Hattie, si è anche dedicato a implementare dispositivi attuativi per coadiuvare crescita degli apprendimenti scolastici in contesti regionali e nazionali con attenzione alle problematiche legate a differenze interculturali. In un momento in cui si discute molto sul ruolo dell'INVALSI e su quali debbano essere le funzioni di un Sistema nazionale di valutazione, l'esperienza condotta in questa direzione da diversi anni da questo autore può rappresentare un interessante benchmark.

Nel mondo delle politiche educative sta ormai assumendo rilevanza crescente un orientamento critico nei confronti di una cultura basata su standard verificati con test che gravano dall'esterno sulla scuola; si orienta l'attenzione alla ricerca di soluzioni più flessibili con un maggiore coinvolgimento degli stessi insegnanti nel ridefinire obiettivi e strumenti di valutazione in ragione delle peculiarità contestuali (diversità socioculturali, bisogni speciali, ecc.).

La strada indicata da Hattie suggerisce di passare da una accountability statica a una dinamica. Le scuole vanno messe nelle condizioni di compiere adattamenti ragionevoli, funzionali ai bisogni locali, con il riconoscimento delle istanze avanzate dalle problematiche dell'inclusione, rispetto a obiettivi e percorsi basati su evidenze e standardizzazioni. Occorre accompagnare l'indicazione di risultati standardizzati con modelli e risorse che aiutino gli insegnanti a definire i loro traguardi in una forma adeguatamente "sfidante», tuttavia nel pieno rispetto delle precondizioni in cui vengono a operare. Un sistema di valutazione deve assumersi l'impegno di rendere immediatamente visibile agli occhi di capi d'istituto e di insegnanti (e soprattutto degli alunni stessi) come concretamente possano essere effettuati gli avanzamenti del proprio apprendimento, facendo percepire subito «il passo in avanti» che la scuola o la singola classe deve compiere. 


\section{RIFERIMENTI BIBLIOGRAFICI}

ACRL (2000). Information literacy competency standards for higher education. http:// www.ala.org/acrl/standards/informationliteracycompetency.

Black, P., \& Wiliam, D. (1998). Inside the black box: Raising standards through classroom assessment. Phi Delta Kappan, 80(2), 139-148.

Bloom, B. S. (1968). Learning for mastery. Evaluation Comment, 1(2), 1-12.

Bonaiuti, G., \& Vivanet, G. (2013). L'utilizzo della Rete per la ricerca di informazioni affidabili. Form@re, Open Journal per la Formazione in Rete, 13(2). http://www.fupress.net/index.php/formare/article/view/13263/0.

Bruni, F., \& Vivanet, G. (2013). Evidence based education: centri di ricerca e risorse in Rete.Form@re, Open Journal per la Formazione in Rete, 13(2). http://www. fupress.net/index.php/formare/article/view/13260.

Calvani, A. (2011a). Principi dell'istruzione e strategie per insegnare. Criteri per una didattica efficace. Roma: Carocci.

Calvani, A. (2011b). «Decision making» nell'istruzione. «Evidence based education» e conoscenze sfidanti. Journal of Educational, Cultural and Psychological Studies, 3. http://www.ledonline.it/index.php/ECPS-Journal/article/view/481.

Calvani, A. (2012). Per un'istruzione evidence based. Analisi teorico metodologica internazionale sulle didattiche efficaci ed inclusive. Trento: Erickson.

Calvani, A. (2013). Evidence based (informed?) education: neopositivismo ingenuo o opportunità epistemologica? Form@re, Open Journal per la Formazione in Rete, 13(2). http://www.fupress.net/index.php/formare/article/view/13259.

Calvani, A., \& Menichetti, L. (2013). Evidence based education: superare il gap tra ricerca e pratica. Form@re, Open Journal per la Formazione in Rete, 13(2). http://www.fupress.net/index.php/formare/article/view/13252.

Clark, R. C., \& Lyons, C. (2010). Graphics for learning: Proven guidelines for planning, designing, and evaluating visuals in training materials. London: John Wiley \& Sons.

Clark, R. C., Nguyen, F., \& Sweller, J. (2011). Efficiency in learning: Evidence-based guidelines to manage cognitive load. London: John Wiley \& Sons.

Coe, R. (1999). A manifesto for evidence-based education. http://www.cem.org/ evidence-based-education/manifesto-for-evidence-based-education.

Davies, P. (1999). What is evidence-based education? British Journal of Educational Studies, 47(2), 108-121. http://www.jstor.org/stable/3122195.

Form@re (2013).Form@re, Open Journal per la Formazione in Rete, 13(2), 13(3), 13(4). http://www.fupress.net/index.php/formare/issue/archive.

Frontline (2013). No child left behind Articles. http://www.pbs.org/wgbh/pages/ frontline/shows/schools/nochild. 
Gruber, K. (2011), Assessing and accommodating learning styles: Is it really a myth? OCWTP, Common Ground, 47. http://www.ocwtp.net/PDFs/Trainer\%20 Resources/CG\%200211.pdf.

Hammersley, M. (Ed.). (2007). Educational research and evidence-based practice. London: Open University Sage Publications.

Hargreaves, H. D. (2007). Teaching as a research based profession: Possibilities and prospects (The Teacher Training Agency Lecture 1996). In M. Hammersley (Ed.), Educational research and evidence-based practice (pp. 3-17), London: Open University Sage Publications.

Hattie, J. (2009). Visible learning: A synthesis of over 800 meta-analyses relating to achievement. London - New York: Routledge.

Hattie, J. (2012). Visible learning for teachers: Maximizing impact on learning. London - New York: Routledge.

Hattie, J. (2013). Di che natura è l'evidenza che fa la differenza per l'apprendimento? Trad. it. di I. Salvadori. Form@re, Open Journal per la Formazione in Rete, 13(2). http://www.fupress.net/index.php/formare/article/view/13254.

Hattie, J., \& Anderman, E. M. (Eds.). (2013). International guide to student achievement. London - New York: Routledge.

Hattie, J., \& Gan, M. (2011). Instruction based on feedback. In R. E. Mayer \& P. A. Alexander (Eds.), Handbook of research on learning and instruction (pp. 249-271). New York - London: Routledge.

Hattie, J., Timperley, H. (2007). The power of feedback. Review of Educational Research, 77(1), 81-112.

Holland, R. (2004). Critics are many, but law has solid public support. School Reform News, 1, March.

Jennings, J., \& Rentner, D. S. (2006). Ten big effects of the «No child left behind Act» on public schools. Phi Delta Kappan, 88(2).

Kuhn, T. S. (1979). La struttura delle rivoluzioni scientifiche. Torino: Einaudi.

Marzano, R. J., Pickering, D. J., \& Pollock, J. E. (2001). Classroom instruction that works. Alexandria, VA: Association for Supervision and Curriculum Development (ASCD).

Mayer, R. E. (Ed.). (2005). The Cambridge handbook of multimedia learning. Cambridge: Cambridge University Press.

Mayer, R. E., \& Alexander, P. A. (Eds.). (2010). Handbook of research on learning and instruction. New York: Taylor \& Francis.

Mitchell, D. (2007). What really works in special and inclusive education: Using evidence-based teaching strategies. London - New York: Routledge.

New Zealand Ministry of Education (2011). The Ministry of Education position paper: Assessment. Learning Media Limited. http://www.minedu.govt.nz/theMinistry/ PublicationsAndResources/AssessmentPositionPaper.aspx. 
Nusche, D., Laveault, D., MacBeath, J., \& Santiago, P. (2012). OECD reviews of evaluation and assessment in education: New Zealand 2011. Paris: OECD. http://dx.doi.org/10.1787/9789264116917-en.

Parents for Choice in Education (2013). 25 years later a nation at risk. http://www. choiceineducation.org/index.php?option=com_content\&view=article\&id=5 $85 \&$ Itemid $=186$.

Parlamento Europeo e del Consiglio (2006). Raccomandazione del Parlamento Europeo e del Consiglio del 18 Dicembre 2006 relativa a competenze chiave per l'apprendimento permanente (2006/962/CE). http://eur-lex.europa.eu/ LexUriServ/LexUriServ.do?uri=OJ:L:2006:394:0010:0018:it:pdf.

Pawson, R., \& Tilley, N. (2004). Realistic evaluation. In S. Matthieson (Ed.), Encyclopaedia of evaluation. Beverly Hills, CA: Sage. http://www.Communitymatters. Com.Au/RE_Chapter.pdf.

Popper, K. R. (1972). Congetture e confutazioni. Bologna: Il Mulino.

Scriven, M. S. (1967). The methodology of evaluation. Chicago, IL: Rand NcNally, Perspectives of Curriculum Evaluation, and AERA monograph series on Curriculum Evaluation, 1.

Slavin, R. E. (2004). Education research can and must address "What works» questions. Educational Researcher, 33(1), 27-28.

Smith, E. (2013). «No child left behind» gets left behind. The Wall Street Journal (U.S. edition), 29 Avril. http://online.wsj.com/article/SB100014241278873 24493704578431252983668668.html.

Thrupp, M., \& White, M. (2013). Research, Analysis and Insight into National Standards (RAINS) project. Final Report: National standards and the damage done. Report commissioned by The New Zealand Educational Institute, Te Riu Roa (NZEI). http://www.waikato.ac.nz/_data/assets/pdf_file/0010/179407/ RAINS-Final-report_2013-11-22.pdf.

UNESCO (2008). ICT competency standards for teachers. http://cst.unesco-ci.org/ sites/projects/cst/default.aspx.

U.S. Department of Education - DoE (2002). No child left behind: A desktop reference. Washington, DC: Education Publications Center.

Vivanet, G. (2013). Evidence based education: un quadro storico. Form@re, Open Journal per la Formazione in Rete, 13(2). http://www.fupress.net/index.php/ formare/article/view/13255.

Vivanet, G. (2014). Che cosè l'evidence based education. Roma: Carocci.

Wylie, C., \& Berg, M. (2013). National standards: What difference are they making? Paper presented at the New Zealand Council for Educational Research (NZARE) Annual Conference, Dunedin, 26-28 November. http://www. nzcer.org.nz/research/publications/national-standards-what-difference-arethey-making. 


\section{RiassunTO}

L'Evidence Based Education (EBE) si sta imponendo come orientamento che pone al centro dell'attenzione il problema della affidabilità della ricerca educativa, il suo statuto scientifico e il rapporto tra ricerca e decisione operativa. Su questultimo versante, meno note sono le influenze che da questo orientamento possono derivare per dar luogo a nuovi sistemi di valutazione dei rendimenti scolastici basati su approcci dinamici, difformi dunque da quelli basati semplicemente sulla comunicazione dei risultati in rapporto a standard generali. Nella prima parte di questo lavoro, presenteremo sinteticamente tale orientamento, i centri di ricerca piu noti, e le problematiche generali che lo caratterizzano. Nella seconda parte, mostreremo come le nozioni di "feedback» e di valutazione formativa, aspetti riconosciuti ormai di grande efficacia per gli apprendimenti individuali, possano fare da riferimento anche per modelli di valutazione e orientamento delle scuole, soffermandoci sull'esperienza neozelandese a cui ha collaborato John Hattie, uno dei massimi rappresentanti contemporanei dell'EBE, il quale suggerisce di passare da una "accountability" statica a una dinamica.

Parole chiave: e-asTTle, Feedback, Istruzione basata su prove di efficacia, Valutazione formativa, Valutazione scolastica. 